\title{
A Review on Data Falsification-Based attacks In Vehicular Ad Hoc Network
}

\begin{abstract}
AUTHOR $\begin{gathered}\text { Sutun Atmed Amalki, } \\ \text { computer science ben }\end{gathered}$
\section{ABSTRACT}

Cooperative Intelligent Transportation System (cITS) is one of loT applications whose purpose is to enhance drive safety and efficiency. Several components constitute cITS including vehicles, roadside units and backend systems. Like many loT applications and systems, cITSs are susceptible to a wide-range of intruding or misbehaving attacks that could be launched by attackers from inside or outside of the network. Once a vehicle is compromised, it can be used to launch several types of attacks against other vehicles and/or components of cITS. They can also be used to send false information and messages to the neighboring vehicles, causing severe complications such as traffic congestions and accidents. Such attacks impede the momentum of the integration of cITS technology with existing infrastructure. In this paper, a comprehensive and deep analysis of the state-of-the-art solutions in intrusion and
misbehavior detection for clTS have been conducted. This paper mainly focuses on the misbehavior detection for cITS have been conducted. This paper mainly focuses on the
data falsification-based attacks that manipulate the mobility data and messages data falsification-based attacks that manipulate the mobility data and messages
shared with the neighboring vehicles as it is more challenging and difficult to identify shared with the neighoritigate. The paper can be of great use for research community to explore more and mitigate. The paper can be of great use for research community to explore more
opportunities and new avenues and propose more robust and effective security opportunities and new avenues and propose more
solutions that protect the potential applications in cITSs.
\end{abstract}

\section{INTRODUCTION}

Although the Internet of Things (IoT) technology plays a crucial rule in facilitating dail life and business, they pose several security challenges and concerns as well. The heterogeneity, internet accessibility and the enormous number of connected devices in IoT render applying traditional security measurements ineffective. Although lo devices vary from each other in several aspects such as functionality, computationa capability, software specifications, and network connectivity, they need to work cooperatively to achieve a shared task. As such a security breach to any of those devices can affect the entire system and hinder it from delivering the require mission. Sevich measures, also called preventive measures, thy to prevent the attacks by procting data and communications by means of cryptography and authentication. Althoush preventive measures can prevent attackers from targeting data and information of other nodes they are unable to stop a faulty or compromised node from sharing manipulated and tampered information with the other nodes. On the other hand, the reactive measures aim to detect the attacks and/or mitigate the failure that the might cause. Intrusion detection is an example of reactive measures that identify the presence of suspicious activities and determines which devices have been presence of
compromised.

\section{LITERATURE REVIEW:}

To safeguard cITS, the security solutions need to detect the attacks first. As such, detection solutions are imperative to notify the defense system about the presence of attack. To detect intrusion and misbehavior attacks against cITS, several solutions collected during the normal operations and/or attacks to build the detection models. Using such data, several statistical, machine learning and artificial intelligence techniques have been utilized to model the normal and attack profiles and calculate the detection thresholds and parameters.

\section{- Intrusion Detection in clTS}

IDS focuses on preventing the attacks launched by attackers from outside of the participating vehicles. These solutions try to identify specific types of attacks such as jamming, replay, and sybil attacks. They are normally applied either globally at the main location in the cITS system like RSUs or locally on the vehicle's level. Like IDSs that work on traditional networks, intrusion detection system on cITSs can work cooperatively such that vehicles can share the knowledge about new and emerging threats among each other.

\section{- Misbehavior Detection In clTS}

\section{1- Data-Centric Misbehavior Detection}

As pointed out previously, data-centric misbehavior detection focuses on the data and (he neighboring vehicles in clTS. By utilizing the correlated packets from different sources, the newly received packet is vetted against several criteria like consistency and plausibility to determine its trustworthiness. Using the consistency check, for instance, the average of the previous speed readings recorded for a vehicle can be used to judge the newly reported speed value. Not only are readings from the same vehicle used to determine the consistency of the new information, but also those coming from the neighboring vehicles. One of the main characteristics of consistency-based detection is its limited reliance on domain knowledge, which makes il easy to design and is it imemed Pausibily, on the other hand, employees a predefined model to verfy whether the received message is in line with the undenlying model. Venicle's speed, for instance, could be verified against the law of physics, which makes it impossible for a vehicle to travel at a speed of 1000 $\mathrm{km} /$ hour, which exceeds the upper limit of the known speed for a moving object.

\section{2- Node-Centric Misbehavior Detection}

Node-centric misbehavior detection assesses the vehicle based on its behavior and trustworthics. For the behavioral aspect, the vehicle and the validity of the format of these messages are observed. The trustworthiness, on the other hand, relies on a vehicle's reputation and the voting to determine whether the vehicle is misbehaving. Voling assumes the majority ar honest. In the study carried out by Zhang, et al. , the trustworthiness was employed to evaluate the vehicle and determine whether it was misbehaving. Such evaluation was carried out in the fog layer or cirs based on both intrinsic and extrinsic factors. Intrinsic factors rely on the information of accidents, engne stalstics, trajectory, and proximity to other vehicles. Principal Component Analysis is utilized to analyze the factors and calculte the trustworthiness of the viche. However, redying on the intrinsic factors might not be suitable in case of advanced attacks that manipulate the vehicle's own data. Those advanced attacks can also manipulate the context surrounding the vehicle by creating a collaborative illusion attack that falsifies driving situation information exchanged between the neighboring vehicles.

\section{FUTURE WORK AND}

\section{RESEARCH DIRECTIONS}

Given the limitations of IDSs and MDSs in cITSs, there is still a need to design and develop more robust and accurate security solutions that take into account the fundamental characterstics of smart vehicular networks like the context dynamicity, comming in solutions need to deal with the sophisticated and massive attacks that try to create majority dishonest nodes and manipulate the driving context in the network. Similarly, such a solution needs to be aware of the false information that the compromised and rogue vehicles could share with neighboring vehicles. This could be achieved by involving robust situational assessment that takes into consideration the change of credibility certain context. In our future rearch, we are roing to build a robust misbehavior detection model that takes into consideration the evasive nature of the sophisticated misbehavior attacks. We will also address the challenge that the advanced attackers pose when they try to carry out cascaded infections in order to create majority dishonest in the road section and deceive the detection mechanisms

\section{CONCLUSION}

Existing security solutions for smart vehicular networks were reviewed. Throughout this survey paper the extant misbehavior detection in cITSs were analyzed to highlight the
different issues related to the detection of misbehavior attacks and how the extant solution deal with them, and the pros and cons of each of these solutions in light of the characteristics of the cITS systems. The nature, characteristics and hierarchy of mobility and safety messages exchanged within the cITS system was elaborated. The different types of the cITS system and their safety and mobility data are explained. The solutions to detect these attacks were discussed. These solutions can be categorized
into Intrusion Detection Systems and Misbehavior Detection Systems. The paper started with a general introduction about the smart vehicular networks and the security concerns in cITSs. A background was then provided to introduce the security threats against cirSs with an overview of the solutions that have been proposed so far. Existing IDSs and MDSs solutions for cliss were then analyzed and elaborated in detail. Based on the analysis, the lessons learned, future work and research directions were discussed. As a future work, this research will be extended by including the implementation of some resured works to show the different parameters and factors that govern developing these solutions and determine potential improvement avenues

\section{REFERENCE}

Almalki, S. A., \& Song, J. (2020). A review on data falsification-based attacks in cooperative intelligent transportation

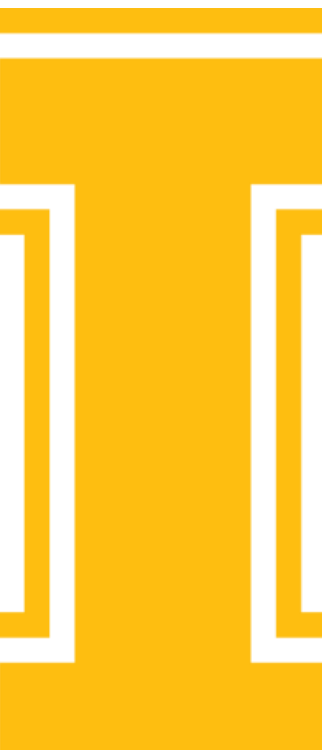

